\title{
The antibiotic resistance crisis, with a focus on the United States
}

\author{
Evan Martens ${ }^{1}$ and Arnold L Demain ${ }^{2}$
}

Beginning with the discovery of penicillin by Alexander Fleming in the late 1920s, antibiotics have revolutionized the field of medicine. They have saved millions of lives each year, alleviated pain and suffering, and have even been used prophylactically for the prevention of infectious diseases. However, we have now reached a crisis where many antibiotics are no longer effective against even the simplest infections. Such infections often result in an increased number of hospitalizations, more treatment failures and the persistence of drug-resistant pathogens. Of particular concern are organisms such as methicillin-resistant Staphylococcus aureus, Clostridium difficile, multidrug and extensively drug-resistant Mycobacterium tuberculosis, Neisseria gonorrhoeae, carbapenem-resistant Enterobacteriaceae and bacteria that produce extended spectrum $\beta$-lactamases, such as Escherichia coli. To make matters worse, there has been a steady decline in the discovery of new and effective antibiotics for a number of reasons. These include increased costs, lack of adequate support from the government, poor returns on investment, regulatory hurdles and pharmaceutical companies that have simply abandoned the antibacterial arena. Instead, many have chosen to focus on developing drugs that will be used on a chronic basis, which will offer a greater profit and more return on investment. Therefore, there is now an urgent need to develop new and useful antibiotics to avoid returning to the 'pre-antibiotic era'. Some potential opportunities for antibiotic discovery include better economic incentives, genome mining, rational metabolic engineering, combinatorial biosynthesis and further exploration of the earth's biodiversity.

The Journal of Antibiotics (2017) 70, 520-526; doi:10.1038/ja.2017.30; published online 1 March 2017

\section{INTRODUCTION}

In 1900, infectious disease was the leading cause of death in the world. ${ }^{1}$ The selective action exerted on pathogenic bacteria and fungi by the 'wonder drugs', that is, microbial secondary metabolites (also known as idiolites), ushered in the antibiotic era which has been of great importance for human beings ever since. Antibiotics are low molecular weight compounds, most of which are natural products made by microorganisms or derived from natural products, which are active at low concentrations against other microorganisms. Some antibiotics such as sulfa drugs and oxazolidinones do not originate from natural products. It is frequently misrepresented that fluoroquinolones are not of natural product origin. Fluoroquinolones are derived from natural products. ${ }^{2,3}$ The first quinolone was derived from the distillation of quinine from the bark of the Cincona tree bark. Therefore, the majority of drugs utilized for chemotherapy against pathogenic microbes are antibiotics.

\section{THE ERA OF ANTIBIOTIC DISCOVERY}

The glorious years of antibiotic discovery, development and production took place in the period between 1940 and the 1960s. Discovery in later years continued but not as rapidly as in the early years. The most important antibiotics include the penicillins, cephalosporins, tetracyclines, aminoglycosides, chloramphenicol, macrolides and glycopeptides. Antibiotics have been crucial in the increase in life expectancy in the United States from 47 years in 1900 to 74 years for males and to 80 years for females in the year $2000 .{ }^{4}$

Over 10000 microbial secondary metabolites have been discovered. ${ }^{5}$ The filamentous bacteria, that is, the actinomycetes, are amazingly prolific in the number of antibiotics which they can produce. About $75 \%$ of known antibiotics are produced by actinomycetes and about $75 \%$ of these are made by a single genus, that is, Streptomyces. Of antibiotics used in medicine, more than $90 \%$ originate from the actinomycetes. In a typical actinomycete, 23-30 gene clusters (about 5\% of the genome) are devoted to secondary metabolism. ${ }^{6}$ Also, important are non-filamentous bacteria, such as species of Bacillus, which can produce over 60 antibiotics. Indeed, $12 \%$ of known antibiotics are produced by non-filamentous bacteria. In addition, some useful antibiotics, such as fusidic acid, are made by fungi (Fusidium coccineum). ${ }^{7}$

\section{UNDERSTANDING THE 'CRISIS'}

Infectious disease is now the second leading killer in the world, number three in developed nations ${ }^{8}$ and fourth in the United States. ${ }^{9}$ Worldwide, 17 million people die each year from bacterial infections. ${ }^{10}$ In the United States, each year approximately two million people are infected with bacteria resistant to antibiotics, of which 23000 will subsequently die as a result of these infections. ${ }^{11}$

${ }^{1}$ Cempra, Inc., Chapel Hill, NC, USA and ${ }^{2}$ Research Institute of Scientists Emeriti (R.I.S.E.), Drew University, Madison, NJ, USA

Correspondence: E Martens, Cempra, Inc., Chapel Hill, NC 27517, USA.

E-mail: emartens@cempra.com

Received 1 November 2016; revised 29 January 2017; accepted 30 January 2017; published online 1 March 2017 
Methicillin-resistant Staphylococcus aureus (MRSA) is responsible for the deaths of 19000 people and 360000 hospitalizations in the United States each year ${ }^{12}$, along with $\$ 3-4$ billion in US healthcare costs. Although MRSA is still a major patient threat, a CDC study published in the Journal of the American Medical Association Internal Medicine showed that invasive (life-threatening) MRSA infections in healthcare settings are declining. ${ }^{13}$ Invasive MRSA infections that began in hospitals declined 54\% between 2005 and 2011, with 30800 fewer severe MRSA infections. In addition, the study also showed 9000 fewer deaths in hospital patients in 2011 versus $2005 .^{13}$

Drug-resistant bacteria kill 25000 people per year in Europe. Perhaps the single biggest public health threat today is antibiotic resistance. ${ }^{14}$ An example is gonorrhea, which was treatable by penicillin in the 1970s, but is becoming resistant even to ceftriaxone, a third generation oral cephalosporins. Gram-negative infections are becoming untreatable due to resistance elements including extended spectrum $\beta$-lactamases and Klebsiella pneumoniae carbapenemase produced by Enterobacteriaciae.

The CDC has categorized the top 18 drug-resistant threats to the United States based on the specific level of concern: urgent, serious and concerning. ${ }^{15}$ Pathogens that are considered urgent threats include Clostridium difficile, carbapenem-resistant Enterobacteriaceae and Neisseria gonorrhoeae. The following pathogens are categorized as serious threats: multidrug-resistant Acinetobacter; drug-resistant Campylobacter; fluconazole-resistant Candida; extended spectrum Enterobacteriaceae; vancomycin-resistant Enterococcus; multidrugresistant Pseudomonas aeruginosa; drug-resistant non-typhoidal Salmonella; drug-resistant Salmonella serotype typhi; drug-resistant Shigella; MRSA; drug-resistant Streptococcus pneumoniae; and drug-resistant tuberculosis (TB). Bacteria which are viewed as concerning threats include vancomycin-resistant S. aureus, erythromycin-resistant Group A streptococcus, and clindamycin-resistant Group B streptococcus. ${ }^{15}$ Other drug resistance problems include: (1) the intestinal bacterium K. pneumoniae becoming resistant to carbapenems; (2) Escherichia coli becoming resistant to fluoroquinolones and causing urinary tract infections and (3) Neisseria gonorrhoeae, the bacterium which causes gonorrhea, becoming resistant to third-generation cephalosporins.

In addition, Clostridium difficile, which is a growing problem in hospitals and long-term care facilities, causes illness in 336000 people in the United States each year and kills 14000 . This represents a $400 \%$ increase in both figures from 2000 to 2013. C. difficile is the leading cause of nosocomial diarrhea around the world and its incidence has markedly increased in the period from 2000 to 2015. ${ }^{16}$ The infections often occur in the hospital after administration of broad-spectrum antibiotics, which deplete the flora of the gut, allowing endogenous or environmental $C$. difficile to grow in the colon. Only three drugs have been approved and used for $C$. difficile infections in the last 30 years, that is, metronidazole, vancomycin and fidaxomicin.

Enteric infections are the fifth leading cause of death worldwide. Nearly $70 \%$ of such infections are food-borne. About 1.5 billion cases of diarrheal disease occur annually, killing 2.2 million people, mainly children. Those under 5 years of age are the most at risk. The infections can be bacterial, viral, parasitic or fungal in etiology. In the United States, there are 48 million cases of food-borne infections with 128000 hospitalizations and 3000 deaths. Nearly 99\% of food-borne infections leading to hospitalization and death are due to 31 known pathogens. These organisms include Brucella spp, Campylobacter spp, Clostridium botulinum, enteroaggressive E. coli, enteropathogenic E. coli, enterotoxigenic E. coli, shigatoxin-producing E. coli, Helicobacter pylori, hepatitis A virus, hepatitis E virus, Listeria monocytogenes, Mycobacterium bovis, Vibrio cholerae, non-cholera Vibrio spp, norovirus, rotavirus, prions, Salmonella spp including Salmonella enterica, Shigella spp, Yersinia spp, and toxins from S. aureus, Clostridium perfringens and Bacillus cereus.

\section{REASONS FOR ANTIBIOTIC RESISTANCE AND HOW TO COMBAT IT}

Resistance is enhanced by the use of antibiotics to promote animal growth and prevent disease in crowded factories and farms. About 30 different antibiotics have been used in food and water for animals. At present, more than $50 \%$ of antibiotics made are used to promote animal growth. In addition, some developing countries provide antibiotics without prescriptions, adding to resistance development.

Antibiotic resistance is due to inactivation by enzymes, such as $\beta$-lactamase, increased efflux of the antibiotic out of cells, decreased uptake of the antibiotic, modification of the target to decrease binding of the antibiotic, amplification of the target, bypassing the essentiality of the target, sequestration of the antibiotic, protection of the target and biofilm formation. ${ }^{17,18}$ A major example of antibiotic resistance is carbapenem-resistant Enterobacteriaceae, ${ }^{19}$ which causes infections of the bladder, lung and blood, which can become life-threatening. The producing organisms are resistant to almost all antibiotics. They were discovered in 1998 but the threat did not become recognized until recently. The carbapenems were developed in the 1980s. Resistant strains of Klebsiella produce an enzyme called KPC (Klebsiella pneumoniae carbapenemase), which breaks down carbapenems. Another enzyme destroying the antibiotic is a metallo-betalactamase. It is found not only in Klebsiella but also in other enterobacteria such as E. coli. Some of the organisms respond to tigecycline and colistin (polymyxin E), but not in all patients.

Community-acquired bacterial pneumonia (CABP) caused by Streptococcus pneumoniae is also becoming a major problem. Resistance is developing against macrolides such as azithromycin and clarithromycin. Macrolide resistance has reached nearly 50\% in the United States. Many strains are not susceptible to pneumococcal vaccines. New and more potent macrolides are greatly needed. ${ }^{20}$ Solithromycin from Cempra, Inc., Chapel Hill, NC, USA, is the only macrolide (fourth generation), which has completed clinical development for community-acquired bacterial pneumonia. The older macrolides inhibit one or two sites on the bacterial ribosome, whereas solithromycin interacts with three distinct sites and is active against multi-drug resistant bacteria, including telithromycin-resistant strains. ${ }^{21}$

A review on overcoming resistance has been published by the staff of The Scientist Journal. ${ }^{22}$ New strategies that are being explored to combat resistance include: (a) modifying old antibiotics into entirely new classes, (b) combining antibiotics, (c) supplementing antibiotics with adjuvants and (d) searching nature for novel antibiotics.

In March of 2015, the US White House issued a plan to cut microbial infections by half within 5 years. ${ }^{23}$ The plan attempts to stop the unnecessary application of antibiotics for growth promotion of farm animals. Unfortunately, it does allow use of antibiotics to help animals survive unsanitary, crowded and stressful confinement conditions. The White House also hopes to fund the discovery of new antibiotics to kill drug-resistant bacteria and the development of diagnostics to rapidly detect them. The plan is called 'the National Action Plan for Combating Antibiotic-Resistant Bacteria' and is focused on C. difficile, carbapenem-resistant Enterobacteriaceae and MRSA, hoping to cut $50 \%$ of such infections by 2020 .

Antibiotic resistance can be overcome in some cases by using combinations of antibiotics. Alternating their application ('alternating 
drug therapy') has been found to slow down the evolution of resistance. Alternating their use rather than combination use may be better, as there would be less drug-drug interaction-related adverse events while it could decrease resistance selection.

Vaccines may also help to lessen the problem. Over one million children with untreated pneumonia die each year. ${ }^{14,24}$ The pneumococcal conjugate vaccine has reduced pneumococcal disease. In the United States, resistant pneumococcal strains decreased by 59\% between 1999 and 2004. However, less than 1/4 of the world's children are protected by pneumococcal conjugate vaccination. A vaccine against $S$. aureus, the most common cause of postoperative infection, has not been successfully produced. Novartis obtained approval in Europe for a vaccine against meningitis. BCG, the bacillus Calmette-Guerin vaccine, is a live but weakened bacterium, which is $80 \%$ effective in healthy children for prevention of TB but it has not been useful in adolescents and young adults who suffer from the pulmonary form of the disease. ${ }^{25}$ Thus, an improved vaccine is urgently needed. In 2011, \$95 million was spent on TB vaccine development and more than 12 vaccines are in clinical trials.

\section{TUBERCULOSIS}

A major problem today is $\mathrm{TB}$, with nine million new cases diagnosed each year and 2.6 million deaths. TB is not only caused by M. tuberculosis but also by Mycobacterium africanum, M. bovis, Mycobacterium caprae, Mycobacterium microti, Mycobacterium pinnipedii and Mycobacteriuim canettii. ${ }^{26}$ Of two billion people who are infected with $M$. tuberculosis, $90 \%$ will not develop symptoms but instead serve as a 'reservoir' for the bacterium, thus enhancing the epidemic. TB therapy now involves a combination of the following four drugs: rifampicin, isoniazid, ethambutol (EMB) and pyrazinamide for 2 months followed by rifampicin and isoniazid for 4 months. New TB drugs are urgently needed for the following reasons: (a) to reduce the duration of therapy; (b) to be effective versus MDR (multidrug-resistant), XDR (extensively drug-resistant) and totally drug-resistant TB strains; (c) to target M. tuberculosis in its latent TB state; (d) to show no antagonism with other anti-TB drugs; and (e) to be compatible with anti-HIV therapy.

Ethambutol is an important agent used to treat TB and is often given in combination with several other drugs. Rifampicins, which include rifampicin, rifapentine, rifabutin and rifalazil, are also used for the treatment of TB. Bedaquiline, a diarylquinoline (initially designated TMC 207 during development), was FDA-approved in 2012, as part of combination therapy for MDR-TB. Additional drugs used against MDR-TB are pyrazinamide, amikacin, kanamycin, capreomycin, cycloserine, ethionamide, ofloxacin, ciprofloxacin, prothionamide and para-aminosalicylic acid (PAS). A new compound, that is, delamanid, was developed by Otsuka company in Tokyo and was approved for use in the European Union. ${ }^{27}$ Currently, the best drugs against Mycobacterium avium complex are clarithromycin, azithromycin and amikacin. They are usually administered with rifampin and ethambutol.

\section{BIOFILMS AND PERSISTERS}

Bacteria forming biofilms, including staphylococci, are very resistant to antibiotics and grow on wounds, scar tissue, medical implants such as joint prostheses, spinal instruments, vascular prosthetic grafts and heart valves. However, they are also important in native infections such as cystic fibrosis, otitis media, endocarditis and urinary tract infections. Antibiotics are not expected to inhibit biofilms but can either inhibit its production or actually penetrate the biofilm. Biofilm infections have been comprehensively reviewed by Bjarnsholt et al. ${ }^{28}$ One way to combat biofilms is to screen compounds against stationary phase planktonic cells and then test them against bacteria growing in biofilms. Such a procedure was used to uncover the anti-biofilm activity of the macrolide azithromycin. Interestingly, exponentially growing $P$. aeruginosa is resistant to azithromycin, whereas stationary phase cells are susceptible. Development of tolerance of organisms to antibiotics takes time, that is, freshly formed biofilms are more susceptible to antibiotics than they are 3 to 5 days later. Mature biofilms can be 1000 times more resistant than planktonic forms to antibiotics and detergents. The best way to eliminate biofilm formation is by prevention, that is, killing the bacteria when they are still planktonic.

Quorum-sensing inhibitors from natural herbal and fungal sources, or chemically synthesized, are capable of regulating expression of many virulence factors of bacteria. However, in vivo experiments have shown that ajoeni (derived from garlic) and the synthetic furanone C-30 block quorum-sensing in P. aeruginosa, inhibit induction of virulence factors and attenuate the otherwise pathogenic organism. Then, the bacteria are phagocytosed and killed. Also, quorum-sensing inhibitors make bacteria more susceptible to conventional antibiotics. Quorum-sensing inhibitors act on both planktonic bacteria and those in biofilms. Ginseng has quorum-sensing inhibitory activity.

Phage therapy is also being considered for control of biofilm infections, either as a preventative or as a curative measure. It has proven effective in vitro and in animal models. Phage cocktails can also eradicate mucoid $P$. aeruginosa biofilms growing on the surface of cystic fibrosis bronchial epithelial cell lines. This indicates that they can penetrate alginate, the major part of the $P$. aeruginosa biofilm matrix.

Metabolic resting states of target pathogenic microbes can allow the organism to survive in the presence of an antibiotic, which kills growing cells. ${ }^{29}$ Such resting states include biofilms, which are formed by Gram-positive staphylococci and streptococci, Gram-negative pseudomonads and metabolically dormant mycobacteria. In such resting states, cell wall synthesis and protein biosynthesis are downregulated and antibiotics acting against such activities may be resisted. Such downregulation may also lead to persisters. In such a state, the cell envelope thickness often increases, making it difficult for the antibiotic to get into the cell. Interestingly, the new diarylquinolines are very active against resting bacteria and eradicate $S$. aureus biofilms. ${ }^{30}$

Persister cells exist in biofilms as a small subpopulation that is highly resistant to killing by antibiotics. Persister cells are antibiotictolerant and are responsible for antibiotic tolerance of biofilms. ${ }^{31}$ They are phenotypic variants of ordinary bacteria. Since antibiotic targets are dormant in persisters, the antibiotics are ineffective against them. Persisters were discovered in 1944 by Bigger. ${ }^{32}$ Conlon et al. ${ }^{33}$ studied acyldepsipeptide (ADEP), an activator of ClpP protease, known to kill growing cells, as a possible killer of persisters. ADEPs are produced by Streptomyces hawaiensis ${ }^{34}$ and a potent derivative, ADEP4 is a good killer of Gram-positive bacteria. ${ }^{35}$ They found that ADEP4-activated protein degradation in non-growing cells and in its presence, ClpP became a fairly non-specific protease, resulting in killing of persisters by degrading over 400 proteins, and forcing the cells to self-digest. The combination of ADEP4 and rifampicin completely eradicated S. aureus biofilms in vitro and in a mouse model of a chronic infection. With rifampicin, it eradicates persisters in growing, stationary and biofilm populations of $S$. aureus in vitro. ${ }^{33}$ 


\section{THE NEED FOR NEW ANTIMICROBIALS}

The global need for new antibiotics has been pointed out by Laxminarayan et al. ${ }^{36}$ They stressed that infectious diseases cause $1 / 5$ th of all deaths around the world each year and are the leading killer of children under 5 years of age. They suggested the following measures: (i) sharing of information about drug discovery among companies and students throughout the world ('open source platforms'); (ii) sharing compound libraries; (iii) providing financial incentives for early development of new antibiotics through preclinical and early clinical trials by governments, charities and international government initiatives; (iv) spreading financial risk between the financial community and new drug developers; and (v) funding international drug discovery and scale-up efforts of small companies in low-income countries and universities studying antibiotic resistance.

\section{REASONS FOR THE DROP-OFF IN DISCOVERY}

One reason is 'merger mania' in the pharmaceutical industry. Mergers in the pharmaceutical industry have decreased the number of groups searching for new antibiotics. As recently as 2009, some major companies underwent mergers, for example, Wyeth with Pfizer, and Schering-Plough with Merck. According to Drews, ${ }^{37}$ who spent many years in the pharmaceutical industry, (i) larger pharmaceutical companies formed by mergers do not become more productive, and (ii) the probability of producing a blockbuster drug is not a linear function of company size. Even many drug executives now realize that mergers can actually have a negative impact on $\mathrm{R} \& \mathrm{D}$ productivity. Nearly 40 major mergers in the pharmaceutical industry took place between 1985 and $2005 .^{38}$

A second reason involves the nature of natural products. Among medicines used up until 1996, 80\% were natural products or inspired by natural products. ${ }^{39}$ Of the 868 new chemical entities approved between 1981 and 2002, 52\% were natural or created around natural product structure. It is quite difficult to discover new natural products with antibacterial activity when those more prevalent in nature have already been discovered. As a result, there has been a misguided loss of interest by companies in natural products, especially those with antibiotic activity. The industry has opted to save funds by eliminating natural product departments or decreasing their relevance in the hunt for new drugs. Large pharmaceutical companies that have dropped or significantly reduced research on discovery of antibiotics include Merck, Wyeth (now part of Pfizer), Aventis, Eli Lilly, GlaxoSmithKline, Bristol-Myers Squibb, Abbott Labs and Proctor and Gamble. ${ }^{40}$

A third reason is the increased costs and the amount of time necessary to put a drug on the market. Clinical development time doubled between 1982 and 2002 to 6 years. This included 1 year of Phase I (involving 20-30 healthy volunteers for safety, tolerability, pharmacokinetics and dosage), 1.5 years for Phase II (100-300 patient volunteers for efficacy and side effects) and 3.5 years for phase III (1000-5000 patient volunteers monitoring adverse effects of long-term use). In addition, there may be 2-10 years for discovery, 4 years for preclinical testing, 1 year of FDA review and approval and 1 year of post-marketing testing. ${ }^{41}$ Although some estimate that the total time to get a drug on the market is $12-15$ years and the costing is $\$ 1.2$ billion, ${ }^{42,43}$ the above breakdown indicates that it could take as long as 14-22 years. However, for anti-infectives, the average number of months in the New Drug Application phase is 24, less than that observed in other therapeutic areas such as cardiovascular (30), NSAID (39) and neuropharmacological (43). ${ }^{44}$

As a result of the increased costs, the pharmaceutical industry's discovery efforts in the 1990s moved away from natural products to combinatorial chemistry followed by high-throughput screening
(HTS). This was done because it was considered that natural product extracts were not amenable to HTS. ${ }^{45}$ Although it was thought that combinatorial chemistry and HTS would yield many new hits and leads, the results were disappointing despite the extraordinary amount of money spent. ${ }^{46,47}$ The problems were that HTS had not been applied to natural product libraries and that combinatorial chemistry had not utilized natural products as scaffolds. ${ }^{48,49}$ This made no sense since the role of combinatorial chemistry, like those of structurefunction drug design and recombinant DNA technology two and three decades ago, was that of complementing and assisting natural product discovery and development, not replacing them. Combinatorial chemistry is great for improving leads but not for the discovery of new leads. However, when combinatorial chemistry is applied to natural products, it could be effective. It is encouraging that natural products are again being used by chemists as combinatorial chemistry scaffolds for synthesis of potential drugs. ${ }^{50}$

Despite the great costs of genomic research, the use of genomics has not had a major effect on antibiotic discovery. After 10 years of bacterial genomics, there were still no promising antibacterial agents on the market or even in clinical testing resulting from genomic studies. ${ }^{51,52}$ Indeed, investments in genomics and HTS have had no effect on the number of products in preclinical development or in Phase I clinical trials. However, instead of downgrading natural product screening, there is real opportunity in incorporating it with HTS, combinatorial chemistry, genomics, proteomics and new discoveries being made in the area of biodiversity.

Baltz ${ }^{53}$ has also proposed several reasons for the lack of discovery of new antibiotics: (a) enzymes essential to the viability of pathogens are not readily 'druggable' (that is, they may not have binding sites for inhibitors, or the compounds cannot gain entry into the cell, or the compounds have poor pharmacological properties), (b) targets are not accessible to in vitro screening (for example, if they are ribosomal or part of nascent peptidoglycan) and (c) chemical libraries lack the molecular complexity of natural antibiotics. According to Baltz, we can no longer depend on pharmaceutical companies alone to come up with new antibiotics. The effort will have to come from medical research by academia in collaboration with biotechnological and pharmaceutical companies.

The drop-off in the rate of discovery may also be attributed to an overemphasis on promotion. The amount spent by the pharmaceutical industry to market, promote and advertise their products in 1991 was $\$ 9.2$ billion. By 2004, the amount was $\$ 25$ billion, mainly due to direct advertising to consumers, free drug samples and salaries for drug representatives.

One reason for companies abandoning the antibiotic area is that these compounds are taken for only a short duration by the patient as compared to drugs for heart disease or high-blood pressure, which are usually taken on a chronic basis.

\section{WHAT CAN BE DONE TO CORRECT THE SITUATION?}

The United States has tried to promote new antibiotic discovery and development via the 2012 GAIN ('Generating Antibiotic Incentives Now') Act. Thirty-nine new antibiotics were granted qualified infectious disease product designation between 2012 and late 2014 . The GAIN Act passed by Congress in 2013 provides priority review and an extra 5-7 years of market exclusivity for qualified products against infectious disease ${ }^{54}$ Fast-track designation is applied for new drugs against GAIN pathogens that include MRSA, VRSA, vancomycin-resistant Enterococci, multidrug-resistant Gram-negatives (Acinetobacter, Klebsiella, Pseudomonas, E. coli) and TB. In May 2011, the FDA approved fidaxomicin (Dificid) for the treatment of 
C. difficile-associated diarrhea. In 2014, three (GAIN) antibiotics were FDA-approved: dalbavancin, tedizolid phosphate and oritavancin, all for bacterial skin infections, including those caused by MRSA. The following year, the Center for Drug Evaluation and Research (CDER, part of FDA) approved Avycaz (ceftazidime-avibactam) for the treatment of complicated intra-abdominal infections and complicated urinary tract infections.

New targets are available for screening natural products. Inhibitors of peptide deformylase and fatty acid biosynthesis, new targets not based on genomics, are in clinical trials. Other targets include lipid A biosynthesis and tRNA synthetases. ${ }^{55}$ Furthermore, major improvements have been made in detection, characterization and purification of small molecules. This has been facilitated by different separation techniques that include TLC, column chromatography, flash chromatography, Sephadex chromatography and HPLC. ${ }^{56}$ Other important non-chromatographic techniques such as immunoassay, phytochemical screening assay and Fourier-transform infrared spectroscopy can also be used to aid in the identification of bioactive compounds.

An enzymatic technique called glycorandomization is now being used to prepare glycoside libraries and to make optimized or novel glycoside antibiotics. Sugars in natural products, such as antibiotics, are usually members of the 6-deoxyhexose family. Over 70 different variants were found in products of bacteria, fungi and plants. Engineering the formation of new secondary metabolites in actinomycetes by glycosylation was reviewed by Salas and Mendez. ${ }^{57}$ Novel deoxysugars can be placed on macrolide antibiotics by combinational biosynthesis. ${ }^{58}$ The presence of glycosidic residues on antibiotics is very important for their activity. ${ }^{59}$

The potential of genomics for discovery of new antibacterials has been discussed. ${ }^{60}$ Genome sequencing revealed many more gene clusters for biosynthesis of secondary metabolites than the number of metabolites known at the time. These 'orphan' biosynthetic pathways ${ }^{61}$ are now being activated by determination of optimum conditions for production. S. coelicolor was known to produce four secondary metabolites but at the time that the genome was sequenced, there were 18 additional biosynthetic pathways identified. Genome sequencing of the marine organism Salinispora tropica revealed a circular genome of 5183331 base pairs. ${ }^{62}$ A large portion $(9.9 \%)$ is devoted to secondary metabolism, greater than ever before seen. It contained genes encoding PKS systems of every known family, non-ribosomal peptide synthases and hybrid clusters.

Like bacteria, fungi have many extra clusters of secondary metabolite biosynthetic genes. ${ }^{63}$ Genome sequencing of eight species of Aspergillus (clavatus, flavus, fumigatus, nidulans, niger, oryzae, terreus and fischeri) showed many more clusters, including PKS and NRPS sequences, than those of secondary metabolites known to be produced by these species. Sequencing of the A. nidulans genome revealed 27 polyketide synthases and 14 non-ribosomal peptide synthases, whereas previously less than 10 biosynthetic gene clusters had been known. ${ }^{64}$

Genome mining involves powerful techniques for discovery of new natural products. ${ }^{65}$ In recent years, a number of additional 'silent' secondary metabolites have been found by genome mining. These include coelichelin ${ }^{66}$ from S. coelicolor, geosmin ${ }^{67}$ from Streptomyces avermitilis and epi-isozizaen ${ }^{68}$, germicidins ${ }^{69}$ and mycothiol $^{70}$ from S. coelicolor. Many new compounds have been isolated from other mined microbes. ${ }^{61}$ Since hundreds of microbial genomes have been sequenced, genome mining offers great promise for the future of drug discovery. Multiple gene clusters encoding secondary metabolite production are common in species of Streptomyces, other filamentous actinomycetes and mycobacteria. ${ }^{71}$ S. coelicolor and $S$. avermitilis contain 20-30 of these clusters. On the other hand, most other bacterial genomes lack them.

Existing bacterial species in nature are estimated to number somewhere between $10^{7}$ and $10^{9} .{ }^{72}$ Less than $0.3 \%$ of soil bacteria and less than $0.00001 \%$ of water-associated bacteria have been grown in common laboratory media. ${ }^{73}$ It has been established that 1.1 million fungal species exist. ${ }^{74}$ Of these, about 14000 species of microfungi are known. However, there actually may be 10 times that amount in nature. ${ }^{75} \mathrm{~A}$ useful review on methods to cultivate uncultivated microbes is that of Vartoukian et al. ${ }^{76}$ Of the 61 known bacterial phyla, only 30 have been cultured. ${ }^{77}$ Methods used to culture some of these organisms include dilute nutrient media and simulated natural environmental conditions. To discover new drugs from such sources, a metagenomic approach is recommended. ${ }^{78,79}$ Recent developments in metagenomics have been reviewed by Simon and Daniel. ${ }^{80}$ Baltz $^{53}$ argued that the lack of new antibiotics can be markedly changed by using high-throughput fermentations, isolating marine actinomycetes, mining genomes to find cryptic pathways and employing combinatorial biosynthesis.

Antibiotics in the clinical pipeline in 2013 were reviewed by Butler et al. ${ }^{81}$ Between 1970 and 1999, antibiotics introduced were analogs of existing drugs with the exception of mupirocin (1985), a topical agent against Gram-positive bacteria. Since 2000, new classes launched included linezolid (an oxazolidinone), daptomycin (cyclic lipopeptide), retapamulin (pleuromutilin) and fidaxomicin (tiacumicin). Unfortunately, all of these act only against Gram-positive pathogens. Of great need are antibiotics that will effectively treat Gram-negative bacterial infections.

Although most of the pharmaceutical industry has discontinued screening for natural product antibiotics, the small biotechnology companies and academics have picked up the slack. ${ }^{82,83}$ Some products in the current pipeline include the following: (i) omadacycline (PTK-0796, Paratek), a broad-spectrum tetracycline (an aminomethylcycline) being tested in Phase III trials against skin and skin structure infections and community-acquired pneumonia. Eravacycline, also known as TP-434, is another tetracycline that is being developed by Tetraphase Pharmaceuticals (Watertown, MA, USA). Two Phase III trials were completed, one for complicated intraabdominal infection (IGNITE 1), which was successful and the other for complicated urinary tract infection (IGNITE 2), which failed. An additional trial is currently underway to support the complicated urinary tract infection indication. Nabriva Therapeutics (Vienna, Austria (headquarters); King of Prussia, PA, USA) is providing BC-3781 in Phase II trials for complicated skin and skin structure infections. (ii) Macrolides/ketolides: solithromycin (CEM-101) is being developed by Cempra, Inc., for community-acquired bacterial pneumonia and has successfully completed two Phase III trials for this indication. (iii) ACHN-490, a next-generation aminoglycoside being developed by Achaogen (San Francisco, CA, USA) for the treatment of complicated urinary tract infections and serious bacterial infections due to carbapenem-resistant Enterobacteriaceae, is currently in Phase III trials.

\section{ADDITIONAL SOLUTIONS FOR THE CRISIS}

Aside from the application of new technologies to solve the antibiotic crisis, there are additional possible remedies. One would involve more government support of small companies and academic institutions attempting to discover new antibiotics. Most of the antibacterials in clinical trials are from small pharmaceutical companies and the biotechnology industry. Yet, equally important is that the 
government encourage large pharmaceutical companies to return to antibiotic discovery. Biomedical Advanced R\&D Authority of the US government has supported antibiotic research in companies such as GSK (\$200 million) and Cempra (solithromycin) (\$75 million) to develop antibiotics. ${ }^{81}$ Other products in the pipeline that are supported by Biomedical Advanced R\&D Authority as part of the Broad Spectrum Antimicrobials Program are plazomicin (Achaogen), carbavance (Medicines Company, Parsippany, NJ, USA), BAL30072 (Basilea, Basel, Switzerland) and eravacycline (Tetraphase). ${ }^{84}$

The NIH proposed the establishment of the National Center for Advancing Translational Sciences (NCATS), which would fund the discovery and early-stage development (including preclinical development) of new, small molecule drugs. A useful analysis of the potential of the NCATS has been written by Reed et al. ${ }^{85}$ The article presents an optimistic view of the possibility of bringing more drugs to market, including antibiotics, which are sorely needed. It is anticipated that the NCATS can bridge the gap between discovery and basic research at universities and its commercial development. The NCATS has teamed up with Pfizer, AstraZeneca and Eli Lilly \& Co. to search for new applications of drugs that have failed in clinical development. ${ }^{86}$ NIH has contributed \$20 million to fund academic efforts to find new applications for these drugs.

New incentives were established in 2012 to help solve the antibiotic crisis. The European Innovative Medicines Initiative started the 'New Drugs 4 Bad Bugs' (ND4BB) project, which involves industry, academia and biotechnology companies. The Innovative Medicines Initiative contributed \$134 million and member companies (AstraZeneca, Basilea, GSK, Janssen R\&D (J\&J) and Sanofi) provided another $\$ 141$ million. ${ }^{87}$

With the increase in resistance to commercial antibiotics, antimicrobial peptides are being considered for commercial production. ${ }^{88}$ They contain from 15 to nearly 50 amino acids, are generally positively charged, are synthesized by the ribosomes and modified posttranslationally. Merck (who acquired Cubist pharmaceuticals) is conducting Phase III trials of its lipopeptide surotomycin (CB-315) against the same bacterium. Another group under study are the peptoids, which have a natural amino acid backbone with synthetic side-chain residues conferring protease resistance and increased hydrophobicity, thus enhancing the membrane permeability.

Also of interest are the bacteriocins. The possibility of using these proteinaceous toxins to help solve the antibiotic crisis has been discussed by Cotter et al. ${ }^{89}$ These are small antibacterial peptides, ribosomally produced by bacteria. They are potent, have low toxicity and exhibit broad or narrow spectrum of activity. Some have mechanisms not shown by antibiotics.

Modification of the microbiome, that is, the trillions of microorganisms in the human body, to improve health has been reviewed by Olle. ${ }^{90}$ These organisms play roles in important biological processes such as metabolism, intestinal homeostasis and development of the immune system. Companies are trying to develop therapies involving the use of live microbes to correct the detrimental effects of microbiome imbalances. Recent work has involved fecal transplantation with live organisms for disease treatment and prevention. It involves lavage of a patient's gut, followed by administration by enema or colonoscopy of a fecal sample from a pre-screened donor, consisting of an undefined composition that contains hundreds of species. Many fecal transplantation studies have focused on $C$. difficile infection, which has become epidemic. This is mainly due to a new antibiotic-resistant strain, NAP1. Clinical trials of fecal transplantation after vancomycin treatment have shown improvement (81-94\%) over vancomycin alone (31\% improvement). Other techniques include the oral application of a single strain or a community of beneficial strains (consortia) to positively influence the human flora of the microbiome. In addition, the FDA approved Merck's bezlotoxumab (Zinplava) to reduce the recurrence of $C$. difficile infection in adult patients receiving antibacterial treatment for this infection.

Lastly, a promising approach to combating bacterial infections is the use of antibacterial monoclonal antibodies. ${ }^{91}$ Abthrax (raxibacumab) was approved in December of 2012. It is produced by GSK and neutralizes toxins made by Bacillus anthracis, the causative agent of anthrax. An earlier monoclonal antibody was Synagis (palivizumab), which was approved in 1998 to prevent respiratory syncytial virus attack in pre-term infants and newborn children at high risk. It was developed by MedImmune, now a part of AstraZeneca. Unfortunately, one of the problems facing the monoclonal antibodies is their high cost when compared to small molecule antibiotics.

\section{DEDICATION}

We are pleased to dedicate this review to Dr Satoshi Omura who is known worldwide as an expert in the field of biotechnology, where his work has focused on the discovery, development and biosynthesis of useful compounds produced by microorganisms. Dr Omura studied at the University of Yamanashi and also at the Tokyo University of Science. As a result, he received a $\mathrm{PhD}$ degree in both pharmaceutical science and chemistry. He has been a professor at the Kitasato University and a leader of the Kitasato Institute for over four decades. Dr Omura was a member of the Editorial Board of the Journal of Antibiotics since 1973, and from 2004 to 2013, he served as the Editorin-Chief. Amazingly, he has authored over 1000 publications, including scientific papers, books and patents. Dr Omura has received innumerable awards and honors, most recent of which was the Nobel Prize in Medicine or Physiology in 2015 (along with Dr William Campbell and Dr Youyou Tu) for his discovery concerning the novel therapeutic agent, avermectin. Together with Merck and Co., he has supplied this drug to Africa, which has revolutionized the treatment of infections caused by roundworm parasites, most specifically river blindness (also known as onchocerciasis).

\section{CONFLICT OF INTEREST}

The authors declare no conflict of interest.

1 Yoneyama, H. \& Katsumata, R. Antibiotic resistance in bacteria and its future for novel antibiotic development. Biosci. Biotechnol. Biochem. 70, 1060-1075 (2006).

2 Heeb, S. et al. Quinolones: from antibiotics to autoinducers. FEMS Microbiol. Rev. 35 247-274 (2011).

3 Fernandes, P. Antibacterial discovery and development-the failure of success? Nat. Biotechnol. 24, 1497-1503 (2006).

4 Lederberg, J. Pathways of discovery: infectious history. Science 288, 287-293 (2000).

5 Hamaki, T. et al. Isolation of novel bacteria and actinomycetes using soil-extract agar medium. J. Biosci. Bioeng. 99, 485-492 (2005).

6 Ikeda, H. et al. Complete genome sequence and comparative analysis of the industrial microorganism Streptomyces avermitilis. Nat. Biotechnol. 21, 526-531 (2003).

7 Christiansen, K. J. Fusidic acid. Antimicrobe. Available at http://www.antimicrobe.org/ d29.asp (accessed 23 January 2017).

8 Kraus, C. N. Low hanging fruit in infectious disease drug development. Curr. Opin. Microbiol. 11, 434-438 (2008).

9 Scott, R. W. Defensin mimetics: nature knows best. Am. Biotechnol. Lab. 27, 16-19 (2009).

10 Butler, M. S. \& Buss, A. D. Natural products - the future scaffolds for novel antibiotics? Biochem. Pharmacol. 71, 919-929 (2006).

11 Centers for Disease Control and Prevention (CDC) Antibiotic/antimicrobial resistance. Available at https://www.cdc.gov/drugresistance/ (accessed 23 January 2017).

12 Walsh, C. T. \& Fischbach, M. A. Squashing superbugs-the race for new antibiotics. Sci. Am. 301, 44-51 (2009). 
13 Centers for Disease Control and Prevention (CDC) Methicillin-resistant Staphylococcus aureus (MRSA) - general information about MRSA in healthcare settings. Available at https://www.cdc.gov/mrsa/healthcare/index.html\#q6 (accessed 24 January 2017).

14 Laxminarayan, R. Antibiotic effectiveness: balancing conservation against innovation. Science 345, 1299-1301 (2014).

15 Centers for Disease Control and Prevention (CDC) Biggest threats. Available at https://www.cdc.gov/drugresistance/biggest_threats.html (accessed 24 January 2017).

16 Ivarsson, M. E., Leroux, J. C. \& Castagner, B. Investigational new treatments for Clostridium difficile infection. Drug Discov. Today 20, 602-608 (2015).

17 Singh, S. B. \& Barrett, J. F. Empirical antibacterial drug discovery - foundation in natural products. Biochem. Pharmacol. 71, 1006-1015 (2006).

18 Davies, J. Resistance redux. EMBO Rep. 8, 616-621 (2007).

19 McKenna, M. Antibiotic resistance: the last resort. Nature 499, 394-396 (2013).

20 Fernandes, P. \& Schranz, J. New macrolides essential to treat CABP. J. Gen. Eng. Biotechnol. News 31, 54-55 (2011).

21 Cain, C. ClpPing Persistence. SciBX 6 (2013).

22 The Scientist staff. Overcoming resistance. Scientist 28, (2014).

23 Erickson, B. House clears chemicals bill. Chem. Eng. News 93, 6 (2015).

$24 \mathrm{Ejim}, \mathrm{L}$. et al. Combinations of antibiotics and non-antibiotic drugs enhance antimicrobial efficacy. Nat. Chem. Biol. 7, 348-350 (2011).

25 DeWeerdt, S. Vaccines: an age-old problem. Nature 502, 58-59 (2013).

26 Zumla, A., Nahid, P. \& Cole, S. T. Advances in the development of new tuberculosis drugs and treatment regimens. Nat. Rev. Drug Discov. 12, 388-404 (2013).

27 Maxmen, A. Drug development: a combined effort. Nature 502, 54-56 (2013).

28 Bjarnsholt, T. et al. Applying insights from biofilm biology to drug development-can a new approach be developed? Nat. Rev. Drug Discov. 12, 781-808 (2013).

29 Bald, D. \& Koul, A. Advances in discovery of new antibacterials for combating metabolically resting bacteria. Drug Discov. Today 18, 250-255 (2013).

30 Balemans, W. et al. Novel antibiotics targeting respiratory ATP synthesis in Grampositive pathogenic bacteria. Antimicrob. Agents Chemother. 56, 4131-4139 (2012).

31 Spoering, A. L. \& Lewis, K. GlpD and PIsB participate in persister cell formation in Escherichia coli. J. Bacteriol. 183, 6746-6751 (2001).

32 Bigger, J. W. Treatment of staphylococcal infections with penicillin by intermittent sterilization. Lancet 244, 497-500 (1944).

33 Conlon, B. P. et al. Activated ClpP kills persisters and eradicates a chronic biofilm infection. Nature 503, 365-370 (2013).

34 Michel, K. H. \& Kastner, R. E. A54556 antibiotics and process for production thereof. US Patent 4492650 (1985).

35 Broetz-Oesterhelt, H. et al. Dysregulation of bacterial proteolytic machinery by a new class of antibiotics. Nat. Med. 11, 1082-1087 (2005).

36 Laxminarayan, R. et al. UN high-level meeting on antimicrobials - who do we need? Lancet 388, 218-220 (2016).

37 Drews, J. Quo vadis, biotech? (Part 2). Drug Discov. Today 6, 21-26 (2001).

38 Daemmrich, A. A. \& Bowden, M. E. A rising drug industry. Chem. Eng. News 83, 28-42 (2005).

39 Harvey, A. L. Natural products as a screening resource. Curr. Opin. Chem. Biol. 11, 480-484 (2007).

40 Barrett, J. F. Can biotech deliver new antibiotics? Curr. Opin. Microbiol. 8, 498-503 (2005).

41 Ernst \& Young The Ernst \& Young Fifteenth Annual Report on the Biotechnology Industry (Ernst \& Young LLP, Palo Alto, CA, USA, 2000).

42 Watkins, K. J. Fighting the clock. Chem. Eng. News 80, 27-33 (2002).

43 Burrill, G. S. Personalized medicine or block-busterology? BioPharm 15, 46-50 (2002).

44 Bush, K. Antibacterial drug discovery in the 21st century. Clin. Microbiol. Infect. 10 (suppl 4), 10-17 (2004).

45 Fox, S., Farr-Jones, S. \& Yund, M. A. New directions in drug discovery. Gen. Eng. News $19,10,36,56,66,80$ (1999).

46 Ausman, D. J. Screening's age of insecurity. Modern Drug Discov. 4, 32-34 (2001).

47 Horrobin, D. F. Realism in drug discovery - could Cassandra be right? Nat. Biotechnol. 19, 1099-1100 (2001).

48 Kingston, D. G. \& Newman, D. J. Mother nature's combinatorial libraries: their influence on the synthesis of drugs. Curr. Opin. Drug Discov. Dev. 5, 304-316 (2002).

49 Waldmann, H. \& Breinbauer, R. Nature provides the answer. Screening 6, $46-48$ (2002).

50 Newman, D. J. Natural products as leads to potential drugs: an old process or the new hope for drug discovery? J. Med. Chem. 51, 2589-2599 (2008).

51 Shlaes, D. M., Projan, S. J. \& Edwards, J. E. Jr Antibiotic discovery: state of the state. ASM News 70, 275-281 (2004).

52 Coates, A. R. \& Hu, Y. Novel approaches to developing new antibiotics for bacterial infections. Br. J. Pharmacol. 152, 1147-1154 (2007).

53 Baltz, R. H. Renaissance in antibacterial discovery from actinomycetes. Curr. Opin. Pharmacol. 8, 557-563 (2008).

54 Cain, C. in: Antibiotic Resistance, SciBx Special Issue on Antibiotic Resistance. 2-4 (2013).

55 Bush, K. Why it is important to continue antibacterial drug discovery. ASM News 70 , 282-287 (2004)

56 Sasidharan, S., Chen, Y., Saravanan, D., Sundram, K. M. \& Yoga, L. L. Extraction, isolation and characterization of bioactive compounds from plants' extracts. Afr. J. Tradit. Complement. Altern. Med. 8, 1-10 (2011).
57 Salas, J. A. \& Mendez, C. Engineering the glycosylation of natural products in actinomycetes. Trends Microbiol. 15, 219-232 (2007).

58 Pageni, B. B. et al. Genetically engineered biosynthesis of macrolide derivatives including 4-amino-4,6-dideoxy-L-glucose from Streptomyces venezuelaeYJ003OTBP3. J. Microbiol. Biotechnol. 18, 88-94 (2007).

59 Kren, V. \& Rezanta, T. Sweet antibiotics - the role of glycosidic residues in antibiotic and antitumor activity and their randomization. FEMS Microbiol. Rev. 32, 858-889 (2008).

60 Mills, S. D. When will the genomics investment pay off for antibacterial discovery? Biochem. Pharmacol. 71, 1096-1102 (2006).

61 Gross, H. Strategies to unravel the function of orphan biosynthesis pathways: recent examples and future prospects. Appl. Microbiol. Biotechnol. 75, 267-277 (2007).

62 Udwary, D. W. et al. Genome sequencing reveals complex secondary metabolome in the marine actinomycete Salinospora tropica. Proc. Natl Acad. Sci. USA 104, 10376-10381 (2007).

63 Zhang, H., Wang, Y. \& Pfeifer, B. A. Bacterial hosts for natural product production. Mol. Pharm. 5, 212-225 (2008).

64 Chiang, Y. M. et al. Molecular genetic mining of the Aspergillus secondary metabolome: discovery of the emericellamide biosynthetic pathway. Chem. Biol. 15, 527-532 (2008)

65 Zerkly, M. \& Challis, G. L. Strategies for the discovery of new natural products by genome mining. ChemBioChem 10, 625-633 (2009).

66 Lautru, S., Deeth, R. J., Bailey, L. M. \& Challi, G. L. Discovery of a new peptide natural product from Streptomyces coelicolor genome mining. Nat. Chem. Biol. 1, 265-269 (2005).

67 Cane, D. E. et al. Geosmin biosynthesis in Streptomyces avermitilis. Molecular cloning, expression and mechanistic study of the germacradienol/geosmin synthase. J. Antibiot. 59, 471-479 (2006)

68 Lin, X., Hopson, R. \& Cane, D. E. Genome mining in Streptomyces coelicolor: molecular cloning and characterization of a new sesquiterpene synthase from S. coelicolor. J. Am. Chem. Soc. 128, 6022-6023 (2006).

69 Song, L. et al. Type III polyketide synthase beta-ketoacyl-ACP starter unit and ethylmalonyl-CoA extender unit selectivity discovered by Streptomyces coelicolor genome mining. J. Am. Chem. Soc. 128, 14754-14755 (2006).

70 Park, J. H., Cha, C. J. \& Roe, J. H. Identification of genes for mycothiol biosynthesis in Streptomyces coelicolor A3. J. Microbiol. 44, 121-125 (2006).

71 Busti, E. et al. Antibiotic-producing ability by representatives of a newly discovered lineage of actinomycetes. Microbiology 152, 675-683 (2006).

72 Schloss, P. D. \& Handelsman, J. Status of the microbial census. Mol. Biol. Rev. 68, 686-691 (2004).

73 Amann, R. I., Ludwig, W. \& Schleifer, K. H. Phylogenetic identification and in situ detection of individual microbial cells without cultivation. Microbiol. Rev. 59, 143-169 (1995).

74 Mueller, G. M. et al. Global diversity and distribution of macrofungi. Biodivers. Conserv. 16, 37-48 (2007).

75 Hawksworth, J. Mushrooms: the extent of the unexplored potential. J. Med. Mushrooms 3, 333-337 (2001)

76 Vartoukian, S. R., Palmer, R. M. \& Wade, W. G. Strategies for culture of 'unculturable' bacteria. FEMS Microbiol. Lett. 309, 1-7 (2010).

77 Hugenholtz, P., Hooper, S. D. \& Kyrpides, N. C. Focus: synergistetes. Environ. Microbiol. 11, 1327-1329 (2009).

78 Lorenz, P. \& Eck, J. Metagenomics and industrial applications. Nat. Rev. Microbiol. 3, 510-516 (2005).

79 Lefevre, F. et al. Drugs from hidden bugs: their discovery via untapped resources. Res. Microbiol. 159, 153-161 (2008).

80 Simon, C. \& Daniel, R. Achievements and new knowledge unraveled by metagenomic approaches. Appl. Microbiol. Biotechnol. 85, 265-276 (2009).

81 Butler, M. S., Blaskovich, M. A. \& Cooper, M. A. Antibiotics in the clinical pipeline in 2013. J. Antibiot. 66, 571-591 (2013).

82 Jabes, D. The antibiotic R\&D pipeline: an update. Curr. Opin. Microbiol. 14, 564-566 (2011).

83 Kneller, R. Importance of new companies for drug discovery: origins of a decade of new drugs. Nat. Rev. Drug Discov. 9, 867-882 (2010).

84 U.S. Department of Health \& Human Services, Public Health Emergency. Jarrett, E BARDA Broad Spectrum Antimicrobials (BSA) Program. Available at https://www.phe. gov/ASPRBlog/Lists/Posts/Post.aspx?ID = 97 (accessed 25 January 2017).

85 Reed, J. C. et al. The NIH's role in accelerating translational sciences. Nat. Biotechnol. 10, 16-19 (2012).

86 Jarvis, L. M. NIH initiative aims to partner academics with pharmaceutical companies to revive failed drug candidates. Chem. Eng. News 90, 41-43 (2012).

87 Roberts, J. P. Incentives aim to boost antibiotic development. Nat. Biotechnol. 30 , 735-736 (2012)

88 Fox, J. L. Antimicrobial peptides stage a comeback. Nat. Biotechnol. 31, 379-382 (2013).

89 Cotter, P. D., Ross, P. \& Hill, C. Bacteriocins - a viable alternative to antibiotics? Nat. Rev. Microbiol. 11, 95-103 (2013).

90 Olle, B. Medicines from microbiota. Nat. Biotechnol. 31, 309-315 (2013).

91 Fox, J. L. Anti-infective monoclonals step in where antimicrobials fail. Nat. Biotechnol. 31, 952-954 (2013) 